\title{
La recuperación: servicios que ponen a las personas en primer lugar.
}

Recovery: services that put people first.

\begin{abstract}
Marta Rosillo Herrero a , Mariano Hernández Monsalve ${ }^{\text {b }}$, Simon Peter K. Smith ${ }^{\text {c. }}$
${ }^{a}$ Psicóloga-Coordinadora Centro de Rehabilitación Psicosocial de los Carmenes. Centro propio de la Red Pública de Atención Social a personas con enfermedad mental grave y duradera de la Consejería de Asuntos Sociales de la Comunidad de Madrid. Gestión Técnica Intress. España. ${ }^{b}$ Psiquiatra, Jefe de Servicios de Salud Mental de Tetuán. España. ${ }^{c}$ Psicólogo, Etobicoke Medical Centre Family Health Team, Toronto, Canadá.
\end{abstract}

Correspondencia: Marta Rosillo Herrero (mrosillo@intress.org)

Recibido: 03/08/2012; aceptado: 01/12/2012

RESUMEN: Esta breve revisión pretende recoger los hallazgos fundamentales de la literatura científica referentes al llamado "paradigma" o "modelo" de recuperación en la enfermedad mental. Pasando por la extensión geográfica del concepto en al ámbito Anglo-Sajón, resume resultados de estudios longitudinales y estudios subjetivos y explora los conceptos fundamentales en cuanto las definiciones de la recuperación en términos de valores, resultados y fases. La discusión argumenta por el acercamiento a la recuperación desde los servicios de salud mental y de la rehabilitación psicosocial.

PALABRAS CLAVE: Servicios Comunitarios de Salud Mental, Recuperación, Valores, Trastornos Mentales.
ABSTRACT: This brief review intends to synthesize the fundamental literature regarding the recovery model (or paradigm) in mental health services. Approaching the original English-language literature, this work reviews longitudinal and subjective studies and explores the fundamental concepts of recovery in terms of values, results and phases. The discussion argues for the reorientation of mental health and psychosocial rehabilitation services towards recovery.

KEY WORDS: Community Mental Health Services, Recovery, Values, Mental Disorders.

\section{Introducción.}

En los últimos años, la atención a la salud mental basada en el modelo comunitario se ha visto beneficiada por la perspectiva de la recuperación, que supone reconocer a la persona con enfermedad mental como verdadera protagonista "en primera persona" de su proceso terapéutico y de crecimiento personal (1).

En algunos países (Nueva Zelanda, Canadá, Australia, Inglaterra o Estados Unidos) el concepto de recuperación ha pasado en pocos años a ser protagonista de las políticas de planificación de la atención en salud mental, de la gestión de los servicios, de las prácticas profesionales y está en la raíz de nuevos servicios y redes de atención creadas "por" y "para" personas afectadas de un trastorno mental. 
Pero ¿esta propuesta de recuperación es una verdadera novedad? o más bien ¿no se trata de "descubrir el Mediterráneo" como ironizaba recientemente Onésimo González (2) en esta misma Revista?. La pregunta exige respuestas matizadas, pues si por un lado, es muy cierto que un mínimo rastreo por la dimensión humanizada de la práctica "psico" desde sus raíces más arcaicas llevaría a afirmar que todo este movimiento no es sino humo de un fuego que está en marcha desde hace muchos años, nos parece que no podemos despacharla tan fácilmente. En estos momentos, la propuesta de prácticas centradas en la recuperación va más allá de la anécdota y está produciendo impactos importantes en la actitud de los propios afectados (empoderamiento- involucración de los directamente afectados e interesados, automanejo de la enfermedad, desestigmatización), en la organización de los servicios (participación e implicación de los usuarios del servicio), en la actitud de los profesionales (mayor respeto a la opinión y preferencias de los interesados, cooperación) e incluso obliga a reconsideraciones psicopatológicas (máxima relevancia de la respuesta subjetiva al diagnóstico y a la "enfermedad", papel central de la elaboración del duelo (3) o la relevancia de los factores relacionados con la resiliencia (4), sin olvidar los modelos de crecimiento post-estrés y post-trauma y sus posibles efectos positivos en el ámbito personal tales como: a) crecimiento personal y cambios en la autopercepción; b) promover una orientación más prosocial y valoración del apoyo interpersonal y c) cambios en la filosofía vital y de desarrollo espiritual (5)).

Efectivamente, la idea de recuperación no es nueva en medicina ni en psiquiatría; lo nuevo es la redefinición del término y el carácter innovador con que se presenta, como un modelo alternativo- en lo conceptual y en lo pragmático- frente a las prácticas más rutinarias en los circuitos asistenciales, ya sean servicios convencionales de atención a la salud mental o de rehabilitación psicosocial.

La recuperación es inicialmente un movimiento social que se pone en marcha en el seno de los usuarios de servicios de salud mental, en torno a un conjunto de valores compartidos (implicación personal, autodeterminación, esperanza.....) que se propone como alternativo a la actual situación de valores y perspectivas existentes , y cuya relevancia para los procesos terapéuticos depende tanto de la asunción de esos valores por parte del mundo profesional y de los responsables de la política sanitaria, como del hecho de que "a posteriori" se ha ido disponiendo de una colección de resultados empíricos que demuestran que los tratamientos más eficaces son aquellos "ricos y densos" en ingredientes de "recuperación" (6). 
ORIGINALES Y REVISIONES

El escenario clínico y asistencial al que se propone como alternativa se puede sintetizarse en los siguientes aspectos:

1. Considerar que la psiquiatría actual, y con ella buena parte de la psicología clínica y otras profesiones implicadas en la atención a la salud mental como "tecnologías de lo psico", se ofrecen desde un fuerte profesionalismo, que apenas deja espacio a la dimensión "personal" del afectado.

Frente a las declaraciones y propósitos participativos fundacionales de la salud mental comunitaria, en la práctica predomina la tarea del profesional con funciones de detectar (objetivar) y tratar de modo frecuentemente prescriptivopsicoeducativo, con el riesgo de asumir que la tarea principal del paciente sea "adherirse" al tratamiento (7).

2. Distintas técnicas de rehabilitación psicosocial (RPS) han demostrado su eficacia, pero ninguna en particular produce efectos globales. Siendo tan amplio el panorama de las intervenciones en RPS, y reconociendo que algunas de sus estrategias son próximas a las propuestas de "recuperación", se critican otras prácticas por sus objetivos parciales, o por ser muy directivas (centradas en el tecno-profesionalismo), con poca participación del afectado, y soslayando o pasando a un lugar de sombra la perspectiva personal, biográfica y subjetiva del afectado (8).

3. La preponderancia de modelos basados en síntomas y déficit da protagonismo a consideraciones biologicistas del comportamiento humano que resultan del todo incompletas y desniveladas. Frente a estos modelos de discapacidad se hace necesario cambiar hacia modelos de intervención que enfaticen más el desarrollo de capacidades, competencias y el bienestar de las personas que sufren problemas de salud mental (9). El propio movimiento rehabilitador en sus orígenes apuesta por modelos de competencias donde la visión está centrada en las capacidades, recursos y aspectos positivos de funcionamiento de cada individuo. La recuperación conecta más de cerca con estos enfoques que ponen el énfasis en el cuidado y la promoción de la salud mental, y se ve enriquecida por la perspectiva y aportaciones de la psicología positiva (10).

4. Siendo ineludible la gran heterogeneidad de la esquizofrenia (la clínica, la evolución, su inicio....), la revisión de la evolución (catamnesis de más de 20 años) demuestra que una proporción importante de pacientes han alcanzado cierta recuperación, y un porcentaje menor una recuperación plena (11). Igualmente sabemos hoy que una proporción importante de pacientes presentan remisión de síntomas y que esta remisión (sintomática) y recuperación (funcional) no tiene porque solaparse. No menos inquietante en el escenario de lo clínico es observar como en ocasiones la recuperación parece haberse producido sin necesidad de una intervención profesional o haber sido bastante independiente de la técnica proporcionada por la práctica profesional (una de las razones por las que se propone instalar la 
recuperación en otro paradigma - no tecnológico), y parece más bien depender de otras cuestiones muy directamente relacionadas con la "dimensión humana" y la asunción de valores clave en la relación de ayuda. La eficacia de las intervenciones clínicas (ej:Rehabilitación psicosocial RPS) parece depender no tanto de los componentes específicos de la tecnología aplicada, sino de otros factores no específicos (implicación del afectado en la dirección del proceso, nivel de empoderamiento, presencia de la esperanza en la recuperación....) (12).

Más adelante nos adentraremos en los caminos de la investigación y los datos que diversos estudios científicos arrojan al respecto de estas y otros cuestiones relativas a los procesos de recuperación de una enfermedad mental.

En cuanto a la historia de la construcción y arraigo de esta "vieja novedad", baste por el momento señalar algunos hitos del proceso:

1) Los antecedentes próximos de las distintas formas de autoayuda, desempeño de roles de agentes de ayuda mutua "peer support specialists" (13), transformación de organizaciones (ej: META, es una organización sin ánimo de lucro situada en Phoenix, Arizona) (14) o la implementación de herramientas de autoayuda/autogestión (Wellness Recovery Action Plans -WARP) (15). Pasando por el movimiento de los club sociales, que fueron una referencia emblemática en los inicios de la desinstitucionalización, sirva como ejemplo la Fountain -House, hoy nuevamente de actualidad en los llamados "clubhouse" que funciona a modo de "franquicia" (16), hasta los efectos del movimiento de "vida independiente" de personas con discapacidad ("nada sobre nosotros, sin nosotros") (17), con sus repercusiones en el movimiento de usuarios, y algunas experiencias que se van generalizando de "partnership" (18). Este movimiento produjo uno de sus efectos más relevantes hace muy poco en la convención sobre los derechos de las personas con discapacidad de la Organización de Naciones Unidas. (ONU) (19).

2) La eficacia de algunas prácticas no convencionales alternativas a la hospitalización psiquiátrica clásica (Proyecto Soteria, Windhorse, Dialogo Abierto), basados en el abandono de las medidas coercitivas y la reducción drástica del uso de fármacos, y la apuesta por atmósferas familiares, el cuidado comprensivo y un entorno donde promover la distensión emocional del paciente (20).

3) La reactivación de los rescoldos, el reencendido de algunas experiencias del movimiento anti institucional y de la anti psiquiatría de los años 60 y 70 del siglo pasado ante el predominio de una psiquiatría centrada en el fármaco y en la acción y decisiones de los profesionales $(21,22)$. Aunque sustancialmente la antipsiquiatría es un movimiento alentado y protagonizado por los profesionales, y la recuperación surge entre los propios afectados, ambas experiencias son claros exponentes de la filosofía de la normalización y la defensa de los derechos de los usuarios que promueven la necesidad de que estos participen activa y directamente en procesos de decisión. 
ORIGINALES Y REVISIONES

4) En una línea muy próxima, el prestigio que han ido adquiriendo los movimientos de usuarios de servicios de salud mental (ej. European Network of (ex) users and survivors of psychiatry ENUSP) (23), con una visión crítica sobre el poderío de los servicios centrados en los profesionales, y que apuestan por crear y apoyar nuevos programas alternativos en el sistema psiquiátrico convencional, por ejemplo, "el apoyo mutuo". Toman aquí especial protagonismo la participación de los usuarios en la propia planificación de los servicios. Esta es un área muy destacada actualmente en el movimiento de recuperación y que se materializa en diferentes trabajos como el Proyecto Emilia a nivel Europeo (24) o más cercanamente la experiencia de "Los Agentes de Ayuda Mutua" del Programa de Salud Mental del Servicio Andaluz de Salud, realizada en colaboración con la Federación Andaluza de Asociaciones de Usuarios/as de los servicios de salud mental "En Primera Persona" (1).

5) La relevancia que han ido adquiriendo en los últimos años los "relatos en primera persona" de personas que están en proceso de recuperación, y que muestran la importancia de la perspectiva subjetiva del propio interesado y del entorno próximo en la recuperación; y el que estos relatos hayan saltado a las páginas de revistas relevantes y de prestigio científico como es el "Schizophrenia Bulletin" o, más recientemente, "Psychosis" y otras. Y, en la misma línea, las revelaciones del padecimiento de enfermedad, e informes de la experiencia personal sobre el padecimiento y sobre la percepción de los "ingredientes y factores terapéuticos" por parte de profesionales de salud mental incluyendo psiquiatras y psicólogos , responsables de servicios de salud mental, que han padecido esquizofrenia u otras psicosis y han recibido tratamientos, en ocasiones durante bastantes años, está suponiendo otro aldabonazo en la misma perspectiva $(25,26)$.

6) La existencia de redes que facilitan la recopilación de estas experiencias subjetivas y los estudios sobre los mismos con participación en colaboración de usuarios y profesionales (Ej. Red de escuchadores de voces o Red de trabajo de Recuperación de Escocia). Esta última va generando y recopilando toda una serie de trabajos en investigación y evidencia a favor de la recuperación, sus trabajos recogen experiencias tan interesantes como estudios subjetivos de narrativas de recuperación, la creación y desarrollo de Redes de Apoyo Mutuo o la generación de medidas con un indicador de recuperación (27).

7) El surgimiento de documentos oficiales de salud pública que pretenden operativizar el concepto, con el propósito tanto de trasladarlo a la práctica como hacerlo susceptible de evaluación. Baste señalar la propuesta del Departamento de Salud del Reino Unido (National Institute for Mental Health) (28, 29), que definió las 10 capacidades básicas para orientar los servicios de salud mental hacia la recuperación o la SAMHSA norteamericana (Substance Abuse and Mental Health Services Administration) (30), que en su declaración nacional de consenso estable- 
cen los 10 principio fundamentales que han de guiar la recuperación. Estos y otros documentos están presentes en la generación de políticas sanitarias y organización de los servicios (31), y sirven de base para orientar el cambio asistencial sobre el que nos vamos moviendo.

8) Por último y no menos desdeñable señalar como van surgiendo elaboraciones construidas sobre bases psicopatológicas y empíricas sólidas para asentar este ¿nuevo? concepto, en los edificios de la psicopatología (Ej: duelo, resiliencia, modelo de capacidades, modelos de trauma...) y la psicoterapia (ej: técnicas narrativas, atención plena...), o en la propia rehabilitación (reforzando las líneas previas de rehabilitación centrada en el modelo de competencia) con nuevos ángulos de intervención como puede ser las aportaciones de la psicología positiva y lo que esta supone como plataforma inmejorable para facilitar la recuperación (32).

Sin duda todos estos caminos y muchos otros van marcando territorios que hasta hace no mucho ni siquiera se planteaban en la naturaleza y el tratamiento de los Trastornos Mentales Graves.

\section{Resultados de la investigacion}

Podemos acercarnos a la investigación a través de los estudios longitudinales que pretenden dar cuenta de cuál es el pronóstico real de las personas diagnosticadas con una enfermedad mental crónica EMC. Los estudios subjetivos ponen el énfasis en el significado de la recuperación para el recuperando. También ahondaremos en la búsqueda de medidas operativas que puedan dar cuenta de los resultados del proceso, de los posibles avances y retrocesos habidos. En estas idas y venidas, en estos avances y retrocesos nos podemos parar a observar cuales son los posibles factores que están asociados al éxito o fracaso en la recuperación. Hoy también sabemos que en el proceso de recuperación se pueden describir diversa etapas, que no es un proceso lineal sino continuo, evolutivo.

Estudios Longitudinales: ¿Puede la gente recuperarse de un diagnostico de enfermedad mental?

Las posibilidades de recuperación es un asunto abierto a debate, disponemos de estudios longitudinales con tendencias más pesimistas y otros más optimistas. Uno de los estudios longitudinales más interesantes es el estudio de Vermont (33) que muestra en un seguimiento a 15 años como el $40 \%$ de las personas con un diag- 
ORIGINALES Y REVISIONES

nostico de esquizofrenia muestran periodos de recuperación. Otros estudios y meta análisis cifran en un 40\% las mejorías tras 5 - 6 años (34) y estudios longitudinales promovidos por la OMS cifran también las tasas de recuperación en un $48 \%$ (35). De estos estudios se desprende conclusiones tan sugerentes para la comunidad científica como observar que al menos un $40 \%$ de las personas muestran algún periodo de recuperación y las probabilidades de recuperación se incrementan con el tiempo.

Estudios subjetivos ¿Qué significa la recuperación para la persona en recuperación?

Además es interesante ver como las principales razones que los propios afectados dan para explicar su lucha frente a la recuperación giran en torno a elementos internos tan importantes como la esperanza de cambio y en pensar que hay profesionales, amigos y familiares que creen en ellos.

El incremento en la sensación de control de los propias vidas y el deseo de normalizar la vida plenamente están presente en el discurso espontáneo de los afectados. Se alejan de posiciones centradas en la patología, la enfermedad y los síntomas para acercarse a posiciones que ponen el énfasis en capacidades y en horizontes de desarrollo y crecimiento personal $(25,36)$.

\section{Definición Operacional: ¿Cómo podemos medir un proceso de recuperación?}

Los criterios de mejoría de un trastorno mental han sido en ocasiones más bien raquíticos basados en la mera reducción de síntomas o la disminución de recaídas, los ingresos psiquiátricos han sido en ocasiones los criterios estandarizados para medir la mejoría.

Pero el panorama parece estar empezando a cambiar hacia cotas más altas de exigencia donde criterios funcionales y psicosociales han de estar presentes. Podemos recurrir como definición operacional a los criterios establecidos por Liberman y colaboradores en el 2002 (37).

Supone el desarrollo de la autonomía personal, del desempeño de roles socialmente valiosos, mantener relaciones socioafectivas significativas y en una convivencia con los síntomas que permita a la persona un nivel de integración sociocomunitaria para desarrollar una vida relativamente satisfactoria. 
Tabla 1.

Definición operativa de recuperación en la Esquizofrenia (37) .

\begin{abstract}
Remisión de los síntomas:
Una puntuación de 4 o menos (moderado) en cada uno de los ítems relacionados con síntomas positivos y negativos de la Escala Breve de Evaluación Psiquiátrica durante dos años consecutivos
\end{abstract}

\title{
Funcionamiento ocupacional:
}

Actividad laboral satisfactoria de al menos media jornada en un empleo en el sector competitivo o asistencia satisfactoria de al menos media jornada en una escuela durante dos años consecutivos. Si tiene edad para jubilarse (por ejemplo más de 60 años), una participación activa en actividades lúdicas, familiares o de voluntariado

\section{Vida independiente:}

Vive independientemente sin una supervisión diaria para poder administrar su dinero, hacer la compra, prepara la comida, lavar la ropa, el aseo personal y sin necesitar actividades lúdicas o no laborales estructuradas. Capaz de iniciar sus propias actividades y gestionar su tiempo sin que se lo recuerden familiares ni otros cuidadores. Aunque la mayoría de los individuos viven solos o con un compañero de piso, persona allegada o amigo, algunas personas podrán cumplir este criterio si viven en casa con la familia si se considera que es apropiado dada la cultura o edad. El individuo puede ser beneficiario de pensiones por discapacidad siempre y cuando participe de manera constructiva en actividades instrumentales durante media jornada o más.

\section{Relaciones con sus compañeros}

Asistir a una reunión, acontecimiento social, actividad lúdica, conversación telefónica u otra interacción conjunta con un compañero que no sea un familiar al menos una vez por semana

\section{¿Qué factores están asociados a éxito/fracaso en la recuperación?}

La recuperación es obviamente un camino que conduce a esas meta con mayor o menor fragilidad o transitoriedad. Tradicionalmente, de acuerdo al modelo médico - recuperación clínica, se han considerado como factores asociados a la recuperación el buen nivel de funcionamiento premórbido, la existencia de pocos síntomas negativos o la buena respuesta inicial al tratamiento anti psicótico; como factores comórbidos se han venido apuntando la importancia de la adherencia al tratamiento, el no uso o abuso de sustancias o el buen funcionamiento neurocognitivo entre otros. Pero actualmente empiezan a tomar peso variables psicológicas tan importantes como la esperanza, la sensación de autoeficacia, o la autoestima o incluso como variables más sociales la ausencia de desventaja social (38 - 39). Todas ellos factores sobre los que se puede intervenir psicológica y socialmente. 
ORIGINALES Y REVISIONES

¿Cómo se describe el proceso de recuperación?

Otras líneas de investigación describen la recuperación en términos de un proceso con distintas fases. Andresen y sus colaboradores (40) describen cinco fases progresivas durante las que una persona pasa: desde la moratoria, donde siquiera reconocen posibilidades para cambio, hasta el crecimiento, donde las dificultades están asimiladas en la biografía de la persona y puede crecer como persona más allá de la discapacidad.

La recuperación no es solo un proceso diacrónico, una sucesión de acontecimientos o fenómenos, sino un proceso en el que caben avances y retrocesos en el que se pueden describir etapas, momentos en los que un tipo de intervención u otras pueden resultar más permeables, la recuperación es un proceso continuo, evolutivo y no lineal, es sino vivir creciendo y desarrollándose.

Tabla 2 .

Estudios que recogen las fases de un proceso de Recuperación. (adaptada de Vázquez, Valiente y Smith, 2010 (44))

\begin{tabular}{|c|c|c|c|c|c|}
\hline Fase & $\begin{array}{l}\text { Baxter \& Dile } \\
\text { (41) }\end{array}$ & $\begin{array}{l}\text { Young \& Ensing } \\
\text { (42) }\end{array}$ & $\begin{array}{l}\text { Pettie \& Triolo } \\
\text { (43) }\end{array}$ & $\begin{array}{l}\text { Spaniol, } \\
\text { Wewiorski, } \\
\text { Gagne \& } \\
\text { Anthony (38) }\end{array}$ & $\begin{array}{l}\text { Andresen, Oades } \\
\text { \& Caputi (40) }\end{array}$ \\
\hline 1 & $\begin{array}{l}\text { Crisis: } \\
\text { Recuperación }\end{array}$ & $\begin{array}{l}\text { Iniciar } \\
\text { recuperación }\end{array}$ & $\begin{array}{l}\text { ¿Por qué yo? } \\
\text { Dar sentido a la } \\
\text { enfermedad }\end{array}$ & $\begin{array}{l}\text { Agobiado por la } \\
\text { enfermedad }\end{array}$ & Moratorio \\
\hline 2 & $\begin{array}{l}\text { Decisión: } \\
\text { Reconstruir la } \\
\text { independencia }\end{array}$ & $\begin{array}{l}\text { Reponiendo y } \\
\text { saliendo para } \\
\text { adelante }\end{array}$ & $\begin{array}{l}\text { Y ahora, ¿qué? } \\
\text { Reconstruir la } \\
\text { identidad }\end{array}$ & $\begin{array}{l}\text { Luchando contra } \\
\text { la enfermedad }\end{array}$ & Reconocimiento \\
\hline 3 & $\begin{array}{l}\text { Despertar: } \\
\text { Construir } \\
\text { interdependencia } \\
\text { sana }\end{array}$ & $\begin{array}{l}\text { Mejorando calidad } \\
\text { de vida }\end{array}$ & & $\begin{array}{l}\text { Viviendo con la } \\
\text { enfermedad }\end{array}$ & Preparación \\
\hline 4 & & & & $\begin{array}{l}\text { Viviendo mas allá } \\
\text { de la enfermedad }\end{array}$ & Reconstrucción \\
\hline 5 & & & & & Crecimiento \\
\hline
\end{tabular}

¿Cómo se estructura un servicio para promover la recuperación?

Los cambios positivos no solo deben esperarse en los propios afectados sino que han de estar presentes en instituciones, servicios y profesionales que deben trabajar en instalar unas prácticas basadas en valores positivos.

Farkas (12) realizó un estudio para analizar narrativas de recuperación de personas en recuperación con experiencias en varios tipos de tratamiento, llegando a la conclusión que da igual la modalidad de intervención lo realmente importante es que los valores de recuperación han de estar presentes. 
Para que el concepto de recuperación llegue a ser un hecho necesita de profesionales cuya actitud asistencial esté basada en valores para ofrecer intervenciones no sólo eficaces y que están basadas en la evidencia, sino que también sean percibidas como apropiadas por la propia persona afectada. Elementos importantes en la filosofía asistencial como pueden ser el respeto por el otro, su capacidad de decisión, su perspectiva, la libertad de elección, recobran aquí su protagonismo. A pesar de que hay muchos valores asociados a los servicios orientados hacia la recuperación, existen al menos cuatro valores clave que sostienen el proceso de recuperación y que surgen recurrentemente en la bibliografía acerca de la recuperación y de las experiencias de los propios afectados: orientación hacia la persona, implicación de la persona, autodeterminación y esperanza o potencial de crecimiento.

Tabla 3.

Valores de recuperación (12)

\author{
Orientación hacía la persona \\ Ver la persona en su totalidad, no el problema \\ Centrado en talentos y recursos personales. \\ Aceptación de todos los roles (no solo el de "enfermo") \\ Visión holística, entender la biografía entera.
}

\title{
Implicación de la persona
}

Implicar a la persona en su proceso terapéutico y de cambio

Tomar responsabilidades

Fomentar el sentido de empoderamiento (empowerment) y de identidad propia.

\section{Autodeterminación}

Dejar a la persona tomar decisiones

Ayudar a la persona a retomar el significado de su vida

"Colaborar" en vez de "pautar"

La coacción se convierte en algo contraproducente

\section{Esperanza}

Buscar la oportunidad para crecer en cada circunstancia

Ver y oír que la recuperación es posible.

Asumir el control sobre la propia vida

Potenciar y reconocer cambios positivos

Ir más allá del mantenimiento o la prevención de recaídas

Ser capaz de avanzar y construir espacios vitales en muchos casos nunca conquistados previamente, p.ej.

tener un trabajo, casarse, tener familia, vivir en un piso, tener amigos, etc. 
ORIGINALES Y REVISIONES

En resumen la investigación muestra que la recuperación es posible con el tiempo, representa un proceso - continuo, evolutivo, no lineal, personal - individualizado, a medida, "como yo lo quiero", con significado - nuevo sentido, nuevo marco y superación - no es volver como antes, sino crecer más allá (45).

La recuperación tiene múltiples resultados tanto objetivos como subjetivos más allá de la reducción de síntomas, potencia la persona como agente sanador y como profesional, se puede potenciar mediante conocimientos, valores y destrezas interpersonales

Si la recuperación no es una intervención específica, ni un resultado en concreto, ni un modelo nuevo, ni es lo mismo para todas las personas ¿Qué es entonces?

La recuperación tiene múltiples definiciones. Una de las más extendidas es la propuesta por Anthony que entiende la recuperación como "una manera de vivir una vida satisfactoria, con esperanza y aportaciones aun con las limitaciones causadas por la enfermedad" (46).

Para los profesionales la recuperación es un resultado que se pretende medir a través de objetivos enfocados a fortalecer la autonomía, el desempeño de roles significativos, el fomento de la relación social y el aumento del bienestar.

Para la persona en recuperación es un proceso, es desarrollar un rol valioso, satisfacción con ese rol, estudiante, amigo, miembro de una familia, remisión y control de síntomas de su propio malestar, aumentar sensación de autoeficacia, autonomía, autogobierno de la propia vida y mantener relaciones sociales significativas.

"El objetivo de la recuperación no es llegar a ser "normal". El objetivo es abrazar nuestra vocación humana de llegar a ser más profundamente humano, a pesar de las limitaciones funcionales" (47).

Constituyen metas universales, que se basan también en valores universales.

La perspectiva de recuperación implica enfocar la atención sobre las posibilidades y la mejora respecto a la situación actual, sobre la construcción de un proyecto vital. Ayudar a la gente a recuperar sus vidas y desarrollar al máximo sus propias capacidades como individuos y como ciudadanos y ciudadanas, se convierte así en una de las prioridades principales de los servicios definidos para la atención de personas atendidas en servicios de salud mental y de rehabilitación psicosocial. 


\section{Consideraciones Finales}

El objetivo de este artículo constituía el acercarse al concepto de recuperación más como un propósito que como un modelo, ir más allá de la enfermedad o la superación de síntomas. Plantearse la recuperación como una meta final y deseable, es sin duda todo un reto formidable para los profesionales. Se trata de una evolución, no de una revolución en el entendimiento mismo de la naturaleza de los trastornos mentales, la discusión abierta y crítica de todo lo que implica la recuperación debe ser un punto de apoyo para el crecimiento y el enriquecimiento de la ayuda a prestar a las personas que padecen un problema de salud mental. Como otros paradigmas en la evolución de la salud mental, el paradigma de la recuperación hará reflexionar a todas las personas implicadas (afectados, familias, profesionales, etc.) y pondrá a prueba la metodología y herramientas actualmente en uso.

Abrazar la recuperación significa, en gran parte, que los valores clave estén integrados en cada nivel del servicio y que los procesos de un servicio de rehabilitación o de salud mental se alineen con el proyecto vital a largo plazo de la persona. Requiere la formalización de espacios para la participación significativa de la persona en atención en el desarrollo del servicio y hace necesario que cada profesional lleva a cabo una reflexión profundamente personal acerca de su relación con la persona en atención.

A la vez, la consolidación del paradigma de recuperación en el trabajo en la rehabilitación psicosocial podría permitir la exploración e incorporación de nuevas tecnologías y herramientas para realizar una reorientación. Programas de auto-planificación de ayudas y tratamientos (15), modalidades terapéuticas que inherentemente incorporan valores de recuperación, por ejemplo la Terapia de Aceptación y Compromiso (48), técnicas como mindfulness (49) o bien sistemas de ayuda mutua (entre personas en atención) (1) también podrían incluirse en la implementación de un servicio de rehabilitación psicosocial y servicios de salud mental.

Para llevar a cabo una transformación de los servicios hacia un paradigma de recuperación, además del apoyo institucional, hace falta la habilitación de las personas que utilizan los servicios para ser protagonistas en su propio proceso. Esto se realiza mediante proyectos que a) incluyen a las personas en atención como socios, no como pacientes, b) consolidan valores de recuperación compartidos entre todos y todas y c) aun respetando el significado para las personas directamente implicadas, sean fácilmente extrapolables a otros servicios.

Los cambios positivos no solo deban esperarse en los propios afectados sino en las propias instituciones y profesionales que deban trabajar en instalar una práctica basada en valores positivos (12). 
ORIGINALES Y REVISIONES

\section{BIBLIOGRAFÍA:}

(1) Junta de Andalucía. Consejería de Salud y Bienestar Social. 1 de cada 4 personas padece alguna enfermedad mental a lo largo de su vida. Accesible en: http://www.1decada4.es

(2) González Álvarez, O. El "novedoso paradigma" de la recuperación o el descubrimiento del Mediterráneo. Rev. Asoc. Esp. Neuropsiq.2011;111:529-530.

(3) Chico, M. Impacto emocional de la enfermedad mental. El proceso de duelo como marco para la intervención terapéutica. Burgos:Prosame,2007.

(4) Bonanno, G.A.Loss, Trauma and Human Resilience.American Psychologist.2004;59:20-28.

(5) Páez, D., Vázquez, C., Bosco, S., Gasparre, A., Iraurgi, I., y V. Sezibera. Crecimiento postestrés y post-trauma: posibles aspectos positivos y beneficiosos de la respuesta a los hechos traumáticos. En D. Páez, C. Martín Beristain, J. L. González, N. Basabe y J. De Rivera. Superando la violencia colectiva y construyendo cultura de paz. Madrid: Fundamentos. 2011; p. 311-339.

(6) Slade, M. Personal Recovery and Mental Illness. A guide for mental health professionals. Cambridge University Press, 2009.

(7) Bentall R.P. Medicalizar la mente ¿sirven para algo los tratamientos psiquiátricos?. Barcelona: Herder, 2011.

(8) Hernández Monsalve M. Complejidad, complementariedad e integración de intervenciones en personas con psicosis. En: M. Hernández Monsalve, Pilar Nieto Degregori. Psicoterapia y rehabilitación de pacientes con psicosis. Madrid:Ed Grupo 5, 2012; p. 15-78.

(9) Vázquez, C., Hervás, G. y Ho, S. Intervenciones clínicas basadas en la psicología positiva: fundamentos y aplicaciones. Psicología Conductual. 2006; 14:401-432.

(10) Vázquez, C. y Hervás, G. Salud positiva: Del síntoma al bienestar. En C. Vazquez y G Hervas . Psicología positiva aplicada. Bilbao: Desclee de Brower, 2008; p.18-40.

(11) Jobe, T.H. y Harrow,M. Long-Term Outcome of patients with schizophrenia: A Rewiew. Canadian Journal of Psychiatry. 2005;50:892-900.

(12) Farkas, M., Gagne, C., Anthony, W. \& Chamberlain, J. Implementing recovery oriented evidence based programs: Identifying the critical dimensions. Community Mental Health Journal.2005;41(2): 141-158.

(13) Australia: PEER Support for Mental Health Project. Disponible en: http://www.peersupport. edu.au

(14) Ashcraft, L. \& Anthony, W.A. A Story of Transformation: An Agency Fully Embraces Recovery. Behavioural Healthcare Tomorrow. 2005;14: 12-22.

(15) Copeland, M.E. Guide to Developing a WRAP - Wellness Recovery Action Plan. 2000, 2008. Disponible en: http://www.mentalhealthrecovery.com

(16) Internacional Centre for Club house Development. Disponible en: http://www.iccd.org

(17) http://www.forodevidaindependiente.org

(18) Reino Unido: National Health Services, NHS. Disponible en : http//www.devonpartnership. nhs.uk

(19) Convención de Derechos de los discapacitados de la ONU, Nueva York, 2006. Disponible en: http//www.convenciondiscapacidad.es

(20) Tim Calton, Michael Ferriter, Nick Huband, and Helen Spandler A Systematic Review of the Soteria Paradigm for the Treatment of People Diagnosed With Schizophrenia. Schizophrenia Bulletin. 2008; 34(1): 181-192. 
(21) Lehman, P. Alternativas a la psiquiatría. Rev. Esp. Neuropsiq. 2012;117:137-50.

(22) Bracken P, Thomas P. Postpsychiatry: a new direction for mental health. BMJ 2001; 322: 724-7.

(23) European Network of Users and Survivors of Pschiatry. Disponible en http://www.enusp.org

(24) Palomer.E, Izquierdo. R, Leahy.E, Masferrer.C,Flores.P,El usuario como experto: concepto, modalidades y experiencia desde el Proyecto Emilia. Revista Asociación Española de Neuropsiquiatria, 2010; 105: 109-123.

(25) May, R. Dar sentido a la experiencia psicótica, En Gleeson, J. Intervenciones psicológicas en la psicosis temprana. Bilbao: Desclee de Brouwer, 2005; p. 425-452.

(26) Frese FJ, Knight EL, Saks E. Recovery from schizophrenia: With views of psychiatriscts, psychologist and others diagnostinsed with this disorder. Schizophrenia Bulletin. 2009;35(2): 370-380.

(27) Brown, W. \& Kandrikiriria, N. Recovering mental health in Scotland. Scottish Recovery Network. 2007. Disponible en: http://www.scottishrecovery.net/index.php?option=com docman\&task=cat_view\&gid=58\&dir=DESC\&order=date\&Itemid=120\&limitstart $=25$

(28) Hope, R. 10 Essential Shared Capabilities: A framework for the whole of the mental health workforce. NHS Departement of Health.2004. Disponible en: http://www.dh.gov.uk/en/ Publicationsandstatistics/Publications/PublicationsPolicyAndGuidance/DH_4087169

(29) Samhsa-subbstance abuse and mental health services administration. National consensus statement on mental health recovery. 2004 consensus. Disponible en http://www.samhs.gov

(30) New Freedom Commission on Mental Health Achieving the Promise: Transforming Mental Health Care in America. Final Report. DHHS pub no SMA-03-3832. Rockville Md, Department of Health and Human Services. 2003 Disponible en: www.mentalhealthcommission.gov/reports/finalreport/ fullreport-02.htm

(31) Hernandez Monsalve, M. Perspectivas de futuro. En Pastor, A., Blanco, A., y Navarro, D. Manual de rehabilitación del trastorno mental grave. Madrid; Editorial Síntesis, 2009; p. 713-734.

(32) Vázquez, C. y Nieto, M. Rehabilitación en Salud Mental: viejo problemas y nuevas soluciones. En Pastor, A., Blanco, A., y Navarro, D. . Manual de rehabilitación del trastorno mental grave. Madrid; Editorial Síntesis, 2009; p. 59-74.

(33) Harrow, M., Grossman, L., Jobe, T. \& Herbener, E. Do psychiatric patients ever show periods of recovery? Results from a 15-year multi-follow-up study. Schizophrenia Bulletin 2005; 31 (3): 723-734.

(34) Harding, C., Brooks, G., Ashikaga, T., Strauss, J. \& Breier, A. The Vermont longitudinal study of persons with severe mental illness, II: Long-term outcome of subjects who retrospectively met DSM-III criteria for schizophrenia. Am J Psychiatry. 1987;144:727-735.

(35) Ciompi, L. The natural history of schizophrenia in the long term. The British Journal of Psychiatry136: 413-420.

(36) Chovil, I. Reflections on schizophrenia, learned helplessness /dependence, and recovery. Psychiatric Rehabilitation Journal. 2005; 29(1): 69-71.

(37) Liberman, R., Kopelowicz, A., Ventura, J. \& Gutkind, D. Operational criteria and factors related to recovery from schizophrenia. International Review of Psychiatry. 2002;14:256-272.

(38) Spaniol, L., Wewiorski, N., Gagne, C. \& and Anthony, W. The process of recovery from schizophrenia. International Review of Psychiatry. 2002; 14 (4): 327-336.

(39) Kelley, M. \& Gamble, C. Exploring the concept of recovery in schizophrenia. Journal of Psychiatric Mental Health Nursing. 2005;12:245-251. 
ORIGINALES Y REVISIONES

(40) Andresen, R, Oades, L. \& Caputi,P. The experience of recovery from schizophrenia: towards an empirically validated stage model. Australian and New Zealand Journal of Psychiatry. 2003;37: 586594.

(41) Baxter, E. \& Diehl, S. Emotional stages: Consumers and family members recovering from the trauma of mental illness. Psychiatric Rehabilitation Journal. 1998 ;21: 349-355.

(42) Young, S.L. \& Ensing, D.S. Exploring recovery from the perspective of people with psychiatric disabilities. Psychiatric Rehabilitation Journal. 1999;22(3):219-231.

(43) Pettie, D. \& Triolo, A. Illness as evolution: The search for identity and meaning in the recovery process. Psychiatric Rehabilitation Journal. 1999; 22: 255-262.

(44) Vázquez, C., Valiente, C. \& Smith, S. P. Marco teórico de la evaluación: desde la rehabilitación psicosocial a la recuperación, en: Fernández, A., Touriño, R., Benitez, N., Aelleira, C. Evaluación en Rehabilitación Psicosocial. Valladolid; FEARP,2010;p.15-31.

(45) Townsend W., Boyd, S., Griffin, C. Emerging best practices in mental health recovery. Columbus: Ohio Department of Mental Health, 2003.

(46) Anthony, W.A. Recovery from mental illness: The guiding vision of the mental health service system in the 1990's. Psychosocial Rehabilitation Journal. 1993; 16:11-23.

(47) Copeland, M. WRAP: Wellness Recovery Action Plan. Brattleboro, Vt.: Peach Press, 1997

(48) Hayes, S., Strosahl, K. \& Wilson, K. Acceptance and commitment Therapy: An experiential approach to behaviour change. New York: Guildford Press, 2003.

(49) Kabat-Zinn, J. Full catastrophe living: using the wisdom of your body and mind to face stress, pain and illness. Boston: Delta, 1990. 